\title{
Computational fluid dynamics modelling of the air movement in an environmental test chamber with a respiring manikin
}

Article

Accepted Version

Mahyuddin, N., Awbi, H. B. and Essah, E. A. (2015)

Computational fluid dynamics modelling of the air movement in an environmental test chamber with a respiring manikin.

Journal of Building Performance Simulation, 8 (5). pp. 359374. ISSN 1940-1493 doi:

https://doi.org/10.1080/19401493.2014.956672 Available at https://centaur.reading.ac.uk/37813/

It is advisable to refer to the publisher's version if you intend to cite from the work. See Guidance on citing.

To link to this article DOI: http://dx.doi.org/10.1080/19401493.2014.956672

Publisher: Taylor \& Francis

All outputs in CentAUR are protected by Intellectual Property Rights law, including copyright law. Copyright and IPR is retained by the creators or other copyright holders. Terms and conditions for use of this material are defined in the End User Agreement. 


\section{CentAUR}

Central Archive at the University of Reading

Reading's research outputs online 


\title{
CFD Modelling of the Air Movement in an Environmental Test Chamber with a Respiring Manikin
}

\author{
Norhayati. Mahyuddin ${ }^{1^{*}}$ Hazim B. Awbi ${ }^{2}$ and Emmanuel A.Essah ${ }^{2}$ \\ ${ }^{* 1}$ Faculty of Built Environment, University of Malaya, 50603 Kuala Lumpur, Malaysia; \\ ${ }^{2}$ School of Construction Management and Engineering, University of Reading, RG6 \\ 6AW, United Kingdom.
}

*Corresponding author. Email: hayati@um.edu.my

\begin{abstract}
In recent years, computational fluid dynamics (CFD) has been widely used as a method of simulating airflow and addressing indoor environment problems. The complexity of airflows within the indoor environment would make experimental investigation difficult to undertake and also imposes significant challenges on turbulence modelling for flow prediction. This research examines through CFD visualisation how air is distributed within a room. Measurements of air temperature and air velocity have been performed at a number of points in an environmental test chamber with a human occupant. To complement the experimental results, CFD simulations were carried out and the results enabled detailed analysis and visualisation of spatial distribution of airflow patterns and the effect of different parameters to be predicted. The results demonstrate the complexity of modelling human exhalation within a ventilated enclosure and shed some light into how to achieve more realistic predictions of the airflow within an occupied enclosure.
\end{abstract}

Keywords: computational fluid dynamics; exhalation; airflow distribution, environmental test chamber.

\section{1: Introduction}

In recent years, computational fluid dynamics (CFD) has been widely used as a method of simulating room airflow, studying indoor environment issues and to produce data that may be otherwise difficult to obtain through in-situ measurements. In-situ measurement 
in an enclosed environment generally gives realistic information concerning the air distribution and airflow parameters in the enclosure. In principle, on-site measurements in an enclosed environment (i.e. office, classroom or bedroom) give the most realistic information concerning airflow and air quality. However, due to the variability of outdoor and indoor conditions (i.e. cooling loads, wind speed and directions, water vapour, etc.) which affect the velocity and control of air movement within the measured room, making an estimation using quantitative analysis can be difficult and inaccurate.

To date he investigation of spatial distribution in classrooms due to air movement is ongoing. However, some parameters of high significance (e.g. exhalation generation rate, the production of exhalation mass flow rate, exhalation velocity, etc.) that are needed as boundary conditions in a CFD model are rather difficult to establish because researchers' interpretations of this data vary widely (Shih, Chiu, and Wang 2007, Gao and Niu 2006, Karthikeyan and Samuel 2008, Lin et al. 2011, Melikov and Kaczmarczyk 2007, Sørensen and Voigt 2003). Therefore, most researchers have limited their research towards the study of ventilation effectiveness in indoor environments (Lin et al. 2009, Lu et al. 2010) and the prediction of airborne disease transmission (Gupta, Lin, and Chen 2010, Rim and Novoselac 2009, Gao and Niu 2006). It is sometimes much more important to investigate how the air is distributed into the space rather than to know the total ventilation flow rate (Awbi, 2003). With regards to experimental evaluations, the main parameters for $\mathrm{CO}_{2}$ and airflow measurement strategies involve the positioning of sampling sensors (i.e. the location and height) and how $\mathrm{CO}_{2}$ and air are being distributed in the room have not been considered methodically. This article attempts to address some of these issues incorporating a study involving an environmental test chamber to carry out measurements under controlled conditions. The results from the measurements are then used to validate CFD 
predictions.

\section{2: Methods}

Experimental methods used to investigate the air movement and spatial distribution levels of $\mathrm{CO}_{2}$ in both classrooms and a test chamber provide in-situ data that is useful information for CFD validation (Mahyuddin and Awbi 2010, Mahyuddin 2011). based on this research, Figure 1 illustrates the positioning of sampling devices as used in the experimental chamber with one occupant. This research investigates the potential of a validation test for airflow distribution in enclosed spaces using a combination of CFD predicted data and experimental data obtained from the field work and chamber tests which have been reported elsewhere (Mahyuddin and Awbi 2010). The gradients in temperatures and air speed in the chamber were measured by using 4-wire PlatinumResistance Thermometer (PRT) sensors type K with an accuracy of $\pm 0.15^{\circ} \mathrm{C}$ of reading and Dantec 'Multi-channel Flow analyzer with cables connecting to hot, thin film Omni directional anemometers' This analyser is capable of measuring the air speed at several strategic locations in the chamber within the speed range of $0.05-1.00 \mathrm{~ms}^{-1}$. Both devices collate instantaneous values of air temperature and velocities for the duration of 2 minutes

To verify the accuracy of the CFD simulations using the three different turbulence models, the indoor air velocity and temperature profiles in the chamber were monitored at 13 locations. The positioning of sensors as illustrated in the figure were as follows; (A) left, (B) front, (C) back (D) right and (E) occupant seating position. These locations are assumed to be outside the influence of the buoyant plumes or free convective flow along the walls. However, due to the complexity of indoor airflows, (i.e. low mean air velocity often less than $0.2 \mathrm{~ms}^{-1}$ ) experimental investigations are also 
extremely difficult especially since the accuracy of velocity sensor is with the abovementioned range. In addition, experimental accuracy is also principally determined by the quality of the apparatus used, whereas the accuracy of numerical solutions is dependent on the quality of the discretisations and boundary condition used (Awbi 2003).

The current focus is to examine the methods of simulation that could be used in such a study and establish the most realistic boundary conditions to simulate the air distribution in rooms around occupants as influenced also by the distribution of respiration air.

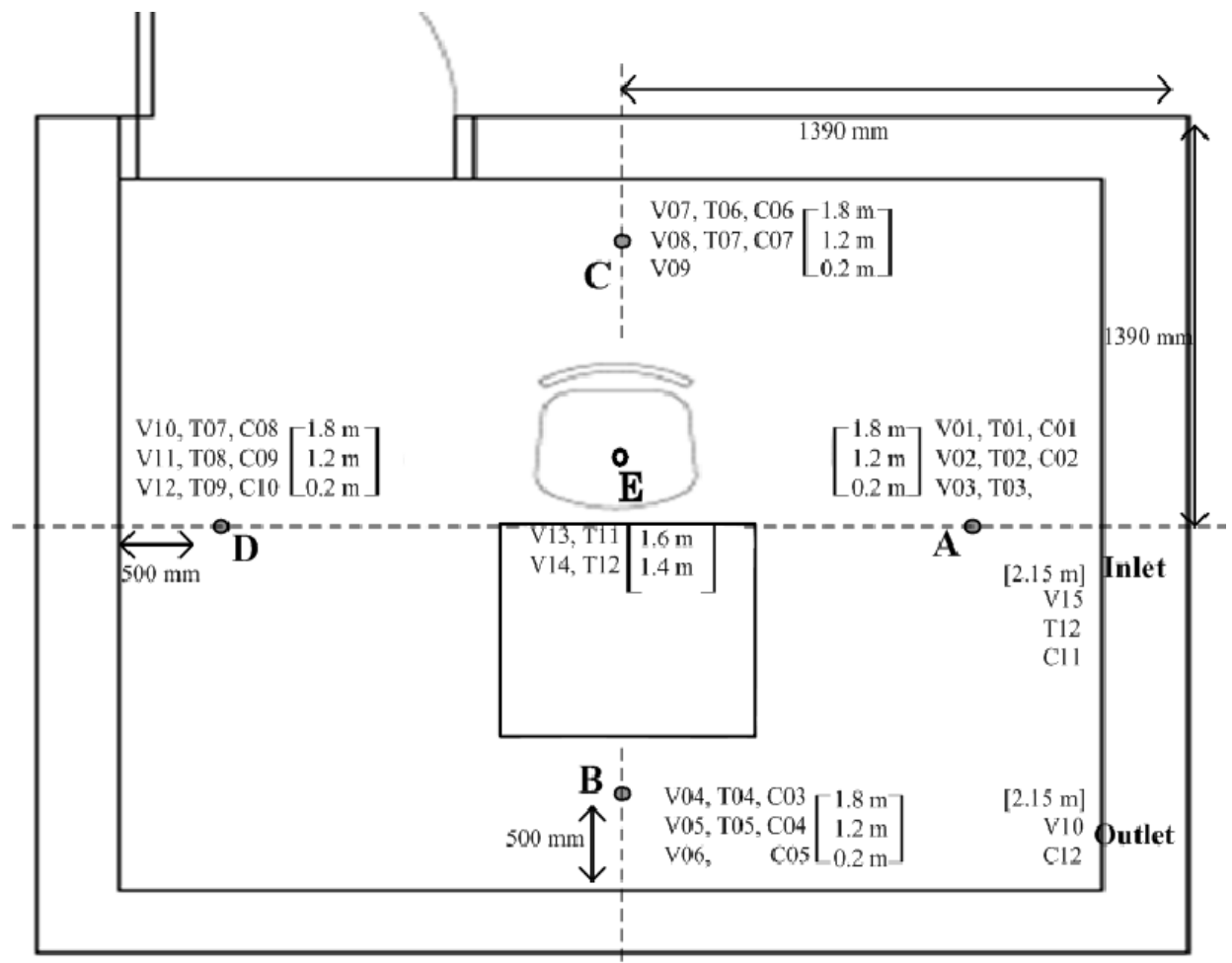

Figure 1 Plan view with positioning of velocity transducers (V), PRT sensors (T) and Carbon Dioxide sensors $(\mathrm{C})$ inside the experimental chamber

The conditions and the modelling of the spatial distributions of airflow in the chamber were modelled using the ANSYS CFX 12.0 code. Initially, the emphasis was on determining the most physically realistic combination of mass and energy transport 
models, fluid properties and boundary conditions that are needed for the modelling. This was necessary to enable the detailed evaluation of the relevant aspects of the internal environment, such as the detailed prediction of airflow parameters within the space which would otherwise have not been possible to determine experimentally alone. It should be noted that the choice of conditions used in the simulations carried out in this research were determined using an environmental test chamber. The discretisation of the computational domain in this research was achieved by means of a mesh that consisted of unstructured grids (i.e. tetrahedral elements) obtained from a mesh generation algorithm based on the automatic mesh in CFX.

To develop a good understanding of the differences between the different CFD turbulence models used, a detailed simulation and analysis of the flow in the chamber was performed. Based on these simulations, various parameters were compared and validated with experimental data in order to assess the extent of significance (deviation from the norm) as well as the trend.

\section{3: Results and Discussion of CFD Validation Test}

The use of CFD involves numerous assumptions, such as the domain size, mesh size, turbulence model, etc. During these modelling tasks, the results of the CFD simulations was evaluated by comparing various turbulence models, boundary conditions and conducting model grid dependency test. The effect of the wall boundary condition, which is one of the most important parameters when investigating the flow patterns within the design domain, (i.e. chamber) was also considered. The method used to represent these conditions in the CFD simulations was also thoroughly investigated. A flow chart of the five modelling strategies incorporated in the simulation is illustrated in Figure 2. 
Step 1-Define Domain size, geometry position and boundary conditions
Step 2-Grid sensitivity test

- Fine mesh

- Medium mesh

- Coarse mesh

\section{Optimum grid for simulations}

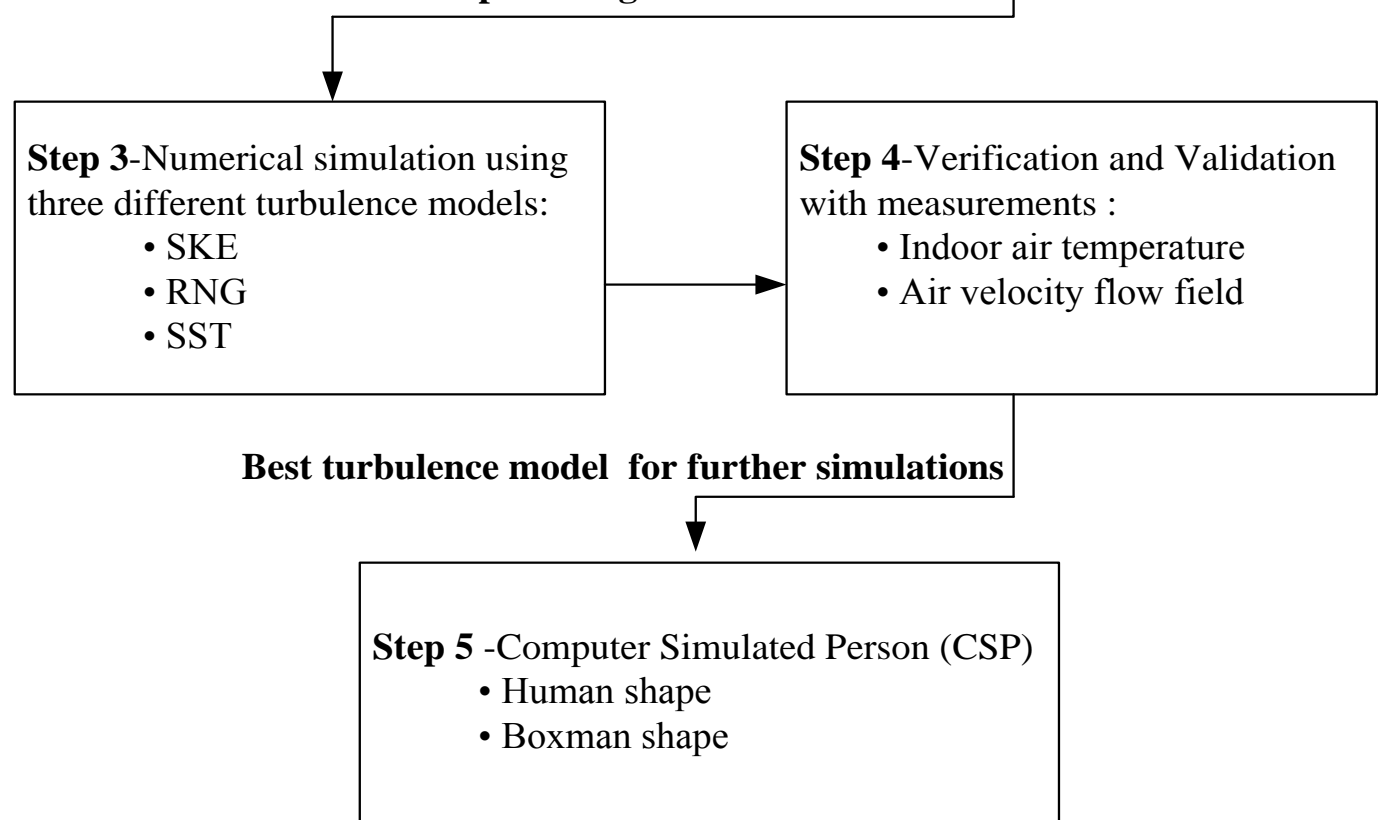

Figure 2 Modelling strategies for computational study.

Considering the fact that this research involves simulations of airflow around a 'human body' within an enclosed environment, the geometry of the computer simulated person (CSP) also plays an important role. The level of complexity involved in this aspect cannot be over emphasised. Various shapes of CSPs ranging from a very detailed human model (manikin) to a simplified 3D shape of a box representation (boxman) were developed and widely adopted in both research and industry to study and provide more insight into thermal comfort issues. These models are roughly put into three categories: simplified shapes (Murakami, Kato, and Zeng 2000, Al-Mogbel 2003, Xing, Hatton, and Awbi 2001), standard human manikins (male and female) (Sørensen and Voigt 2003, Tanabe et al. 2002) and realistic models (Yang et al. 2007, Treeck et al. 2009, Zhang and Yang 2008).However, to identify the best grid (steps 1 and 2) and 
turbulence models (steps 3 and 4) that would be used for further simulations (see Fig. 2), a simple 'boxman' shape CSP is used to minimise the computational time. Although in principle, the closer the computational geometry is to the real geometry of interest the more accurate the predictions are, it is extremely difficult to create a mesh for complex geometry such as that of the 'human being'. Such a domain would also increase the computational time. Therefore, as suggested by Deevy et al (2008) and Topp et al (2002), it is sufficient to approximate the shape of the occupant with a more simple geometry, unless detailed predictions of particle deposition, heat transfer or other quantities very close to the body are needed. In the latter case, a more accurate representation may be necessary.. Therefore, the validation of the CSP (step 5) was only performed once the appropriate grid size and the turbulence model were identified.

In addition, according to Zhang and Chen (2006), Holmes et al. (2000) and Loomans (1998), the most frequently used turbulence model in the simulations of airflow around the human body are the RNG. This is because compared to other turbulence models, they provide more accurate results due to being specifically designed to model low Reynolds number effects and pollutant transport (Chen 1995, Buchanan 1997, Ferziger and Peric 2002). Therefore, prior to choosing the most appropriate turbulence model, the RNG $k-\varepsilon$ model is used to investigate the grid sensitivity test in step 2 .

\section{1: Computational Geometry and Domain (Step 1)}

Defining the computational domain was based on the geometry and flow profile of the model. To obtain more realistic indoor airflow results, a comparison of experimental results with CFD predictions of the airflow distribution close to the human body was carried out inside an environmental chamber. 


\subsection{1: Boundary Condition}

Prediction accuracy is, as with all modelling techniques, highly sensitive to the boundary conditions supplied or assumed by the user (Awbi, 2003, Xu and Chen, 1998, Emmerich, 1997). The types of boundary conditions used in the simulations were wall properties, heat generation, airflow rate at the inlet and the outlet and $\mathrm{CO}_{2}$ production rate by the occupant (Figure 3). However, in Figure 3 objects like legs of tables and chairs supporting the seated CSP are not included to minimise the computational grid size which would reduce computation time.

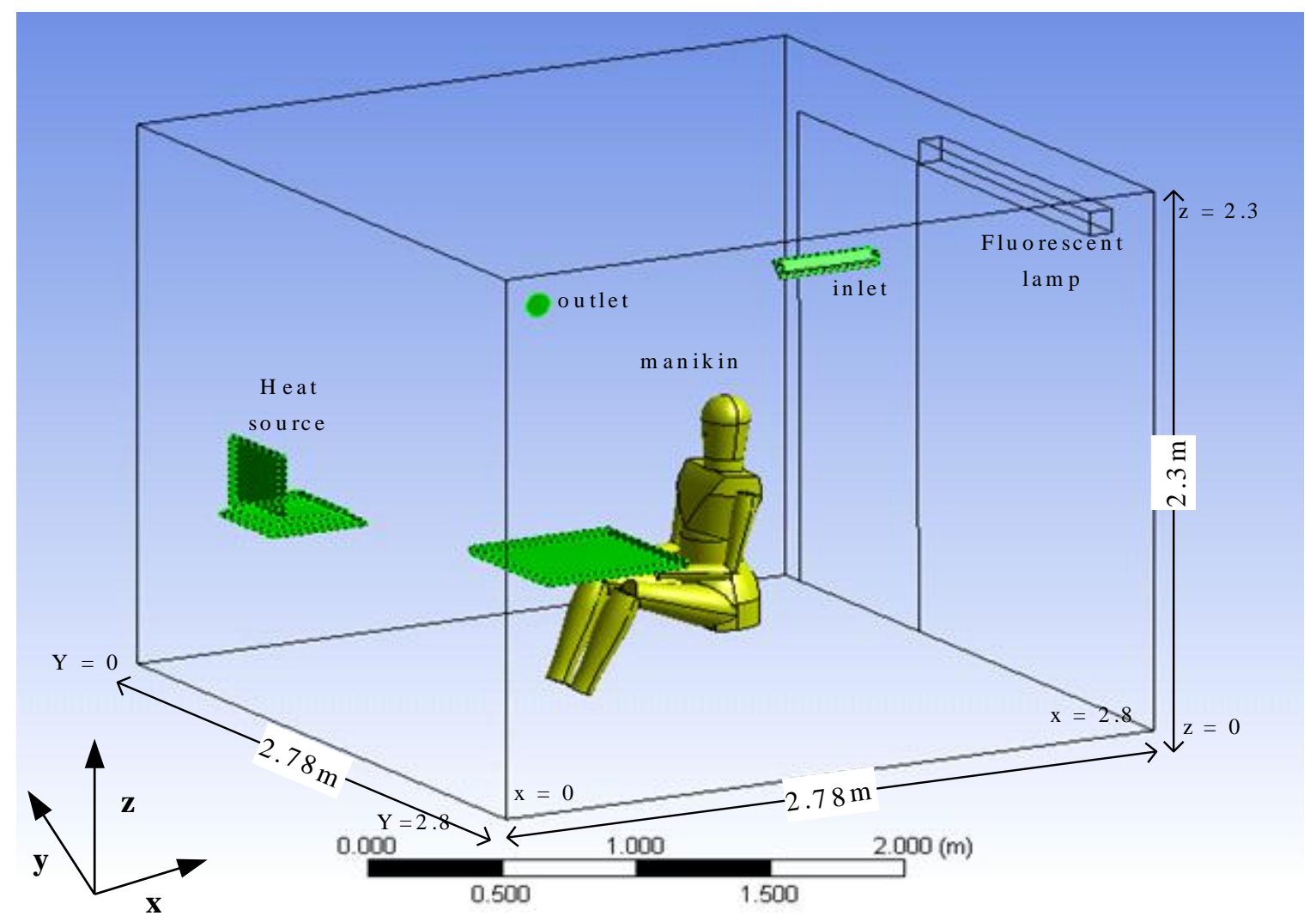

Figure 3 Geometry of experimental chamber

The air inlet was modelled an inlet size of $0.4 \mathrm{~m}$ wide and $0.01 \mathrm{~m}$ high, extending from $\mathrm{x}=1.19 \mathrm{~m}$ to $1.59 \mathrm{~m}$. The diameter of the air outlet from the chamber was $0.1 \mathrm{~m}$ and was positioned at $\mathrm{x}=0.14, \mathrm{y}=0, \mathrm{z}=2.18 \mathrm{~m}$. The dimensions of the table in front of the manikin were $0.6 \mathrm{~m}$ long x $0.6 \mathrm{~m}$ wide and $0.75 \mathrm{~m}$ high. A second 
table with a laptop on it measured $0.3 \mathrm{~m}$ for both length and width and was $0.75 \mathrm{~m}$ high, see Figure 3.

In the middle of the chamber at $\mathrm{x}=1.705$ and $\mathrm{y}=1.08$, a seated CSP with a seated height of $1.2 \mathrm{~m}$ is located. The simulation of nostril exhalation for the CSP is illustrated in Figure 3. $\mathrm{CO}_{2}$ supply (i.e. exhalation) through a nostril of $12 \mathrm{~mm}$ diameter was set as an inlet supply source and was positioned at a height of $1.1 \mathrm{~m}$. In order to apply the boundary conditions in the CFD model it was necessary to create a fluid domain within the confines of the geometry. To allow an increased heat load in the chamber, different heat sources were added. The manikin heat emission was similar to that of a real person. In this study, the thermal comfort and moisture production of the person is not taken into account. Therefore, a mean surface temperature of a human body in the state of physiological thermal neutrality with normal indoor activity of 33.7 ${ }^{\circ} \mathrm{C}$ was used. In addition, a $60 \mathrm{~W}$ laptop located near the front wall and a fluorescent lamp with a reflector located on the back wall was also modelled. The ballast for the fluorescent lamp consumed approximately $36 \mathrm{~W}$. However, due to the reflector casing, the heat flux was difficult to calculate accurately. Therefore, for consistency of the generation output from the heat sources, a surface temperature was used instead of heat flux.

Other boundary conditions were set on the surfaces in the computational domain and are summarized in Table 1. In addition Table 2 lists the locations of the sampling points used for CFD simulations results, which were adopted by the experimental chamber sampling methods (see Figure 1). 
Table 1 Specified values of the numerical methods

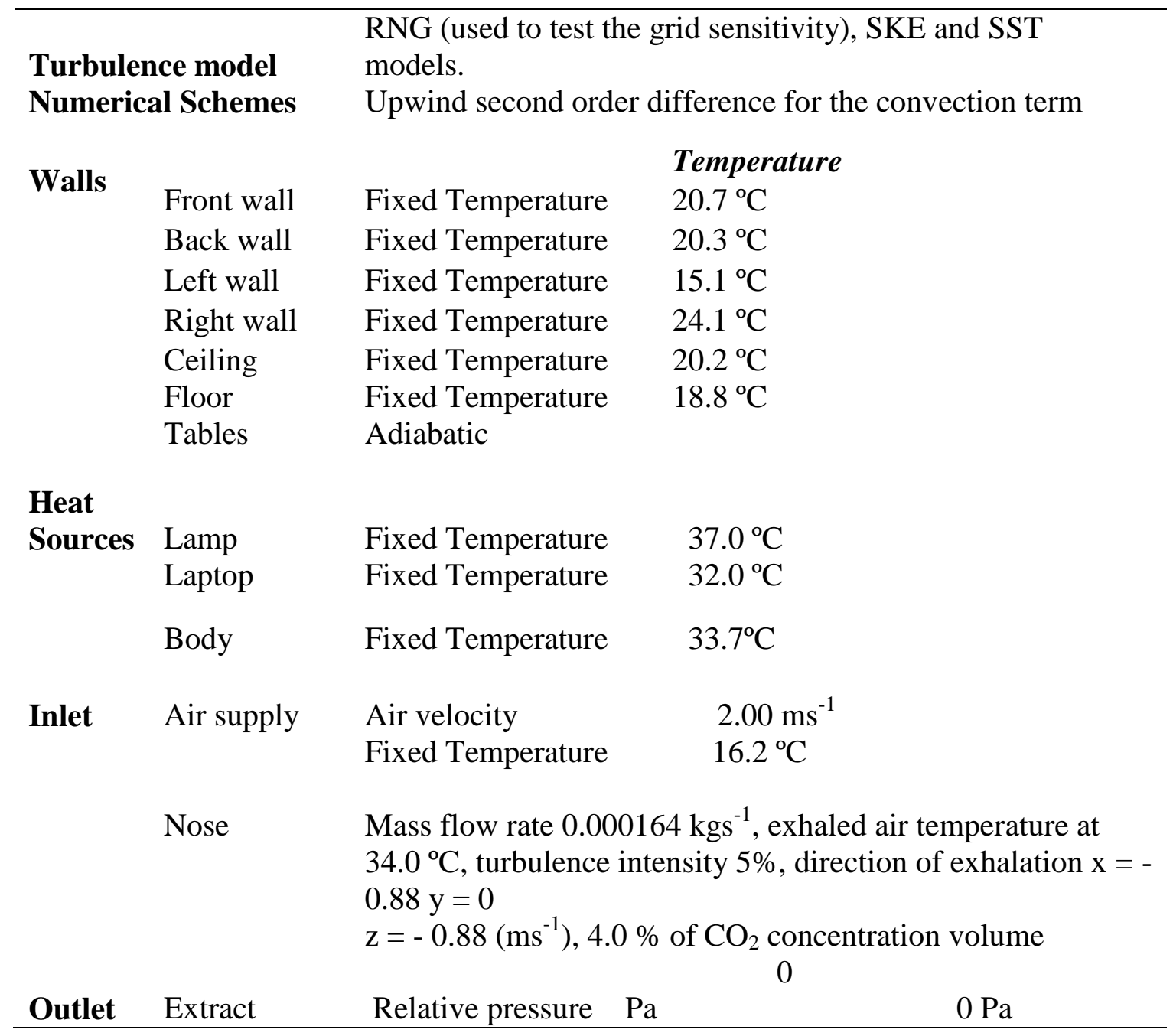

Table 2 Spatial coordinates of sampling sensors in the test chamber

\begin{tabular}{lllll}
\hline \multirow{2}{*}{ Location } & \multicolumn{2}{c}{ Coordinates of Sensor points $(\mathrm{x}, \mathrm{y}, \mathrm{z})$} \\
& $0.2 \mathrm{~m}$ & $1.2 \mathrm{~m}$ & \\
\hline \multirow{2}{*}{ A (Left wall) } & $(0.00,0.50,0.20)$ & $\begin{array}{l}(0.00, \\
1.20)\end{array}$ & 0.50, & \\
& & $(0.50$, & $1.8 \mathrm{~m}$ & $(0.00,0.50,1.80)$ \\
B (Front Wall) & $(0.50,1.39,0.20)$ & $\begin{array}{l}1.20) \\
(2.28,\end{array}$ & & $(0.50,1.39,1.80)$ \\
& & $1.20)$ & & $(2.28,1.39,1.80)$ \\
C (Back Wall) & $(2.28,1.39,0.20)$ & $(1.39$, & 2.28, & \\
& & $1.20)$ & & $(1.39,2.28,1.80)$ \\
\hline
\end{tabular}




\subsubsection{Flow Field with Respiration Process}

With regards to the exhalation flow rate, a nose was modelled on the manikin's body surface (i.e. at the head) and assigned as a respiration inlet boundary condition. This was the emission source of $\mathrm{CO}_{2}$ that was considered as a non-reacting scalar component transported through the flow of air using both convective and diffusive processes. Different descriptions of the breathing process (i.e. the flow rate, frequency of respiration, direction of breathing jet, nose opening, etc.) are discussed in various research (Quatember 2003, Vos et al. 2007, Gao and Niu 2006, Gupta, Lin, and Chen 2010). This research which is also based on research by McArdle et al. (2006), incorporates a person with an average breathing rate of 17 times per minute with a tidal volume $500 \mathrm{ml}$ per breath (McArdle, Katch, and Katch 2006). This value of respiratory frequency was also derived from observations involving 23 adults at a sedentary level which yielded the respiration frequency of $16.74 \pm 0.80 \mathrm{SD}$ (Standard deviation) (Mahyuddin and Awbi 2010). In this research, the exhalation of $\mathrm{CO}_{2}$ from the person was through the nose. Similar values was also used by Gao and Niu (2006) in their research about the human respiration process and transports of air by breathing, coughing and sneezing. Based on Equation 1, the exhaled volume per minute $\left(V_{E}\right)$ during respiration (i.e. $2 \mathrm{~s}$ exhalations and $2 \mathrm{~s}$ inhalations) is derived:

$$
V_{E}=V_{T} \times T_{R}
$$

where $T_{R}$ is the Respiratory period (breath per minute) and $V_{T}$ is the Tidal volume.

To model the manikin exhaling $\mathrm{CO}_{2}$ into the domain, inlet boundary conditions are applied to the surfaces representing the nose of the manikin. The directions of inlet flow velocity, mass flow rate, temperature and the value of total $\mathrm{CO}_{2}$ source in unit of $\mathrm{kg} \mathrm{m}^{-3}$ were specified. Gupta et al., (2010), measured the cross-sectional areas of the nose of 16 individuals ( 8 male and 8 female), and found that the mean area of nose 
opening was $0.71 \pm 0.23 \mathrm{~cm}^{2}$ for male. In the seated boxman designed for this study, a total diameter of $1.2 \mathrm{~cm}$ nostril (i.e. a size of $0.6 \mathrm{~cm}$ in diameter for each nostril) was used. Since only one nostril was used in this study, the nose opening was slightly lower than the result obtained by Gupta et al., (2010), which was $\sim 1.4 \mathrm{~cm}$ ). This difference was considered acceptable, since there is a considerable deviation of nasal geometry in real humans. In addition, the occupant used in the test chamber has a Du Bois area $\left(A_{D}\right)$ of $1.7 \mathrm{~m}^{2}$ (which is smaller than an average adults size $\left(A_{D}=1.8 \mathrm{~m}^{2}\right)$. However, the behaviour of the exhalation jet would depend on its own temperature and momentum, on the temperature of the room and of other interactions, for example the boundary layer flow around a person (Bjorn and Nielsen 2002).

Considering that the flow rate $(\mathrm{Q})$ at a sedentary level is around $8.51 / \mathrm{m}$ over a diameter (D) of $1.2 \mathrm{~cm}$, an exit velocity of $1.25 \mathrm{~m} . \mathrm{s}^{-1}$ at the nostril can be calculated using Equation 2.

$$
V_{e x i t}=\frac{Q}{S}=\frac{Q}{\left(\pi D^{2} / 4\right)}
$$

The results as visualised for a person exhaling through the nose using smoke as the indicator and a manikin in a CFD simulation of an exhalation process are shown in Figure 4. Based on the smoke test carried out in the chamber (Figure 4a), the exhalation jet through the nose was identified to be directed downwards from the nostrils with an angle of approximately $45^{\circ}$ below the horizontal line, which fits well with the observation made by Hyldgaard (1994) and Haselton and Sperandio (1988). The results in Figure $4 \mathrm{~b}$ also show that the numerical simulation of the exhalation jet is also set at a $45^{\circ}$ from the horizontal. It is observed from both figures (Figure 4 a $\&$ b) that the velocity profile of the exhaled air does not continue to be uniform at an angle of $45^{\circ}$. 
The illustrations in Figure 4 demonstrate turbulence effects on the exhaled jet. This is partly due to the air entrainment in the zone of investigation.

In reality, due to the body core temperature (normally about $37.2^{\circ} \mathrm{C}$ ), the inhaled air is exhaled at about $34.0^{\circ} \mathrm{C}$. The velocity vectors for the exhalation region are large in the front side of the human face from the nose to the lower parts, as shown in Figure $4 \mathrm{~b}$. The warm exhalation jet is observed to entrain more surrounding air from the upper region.
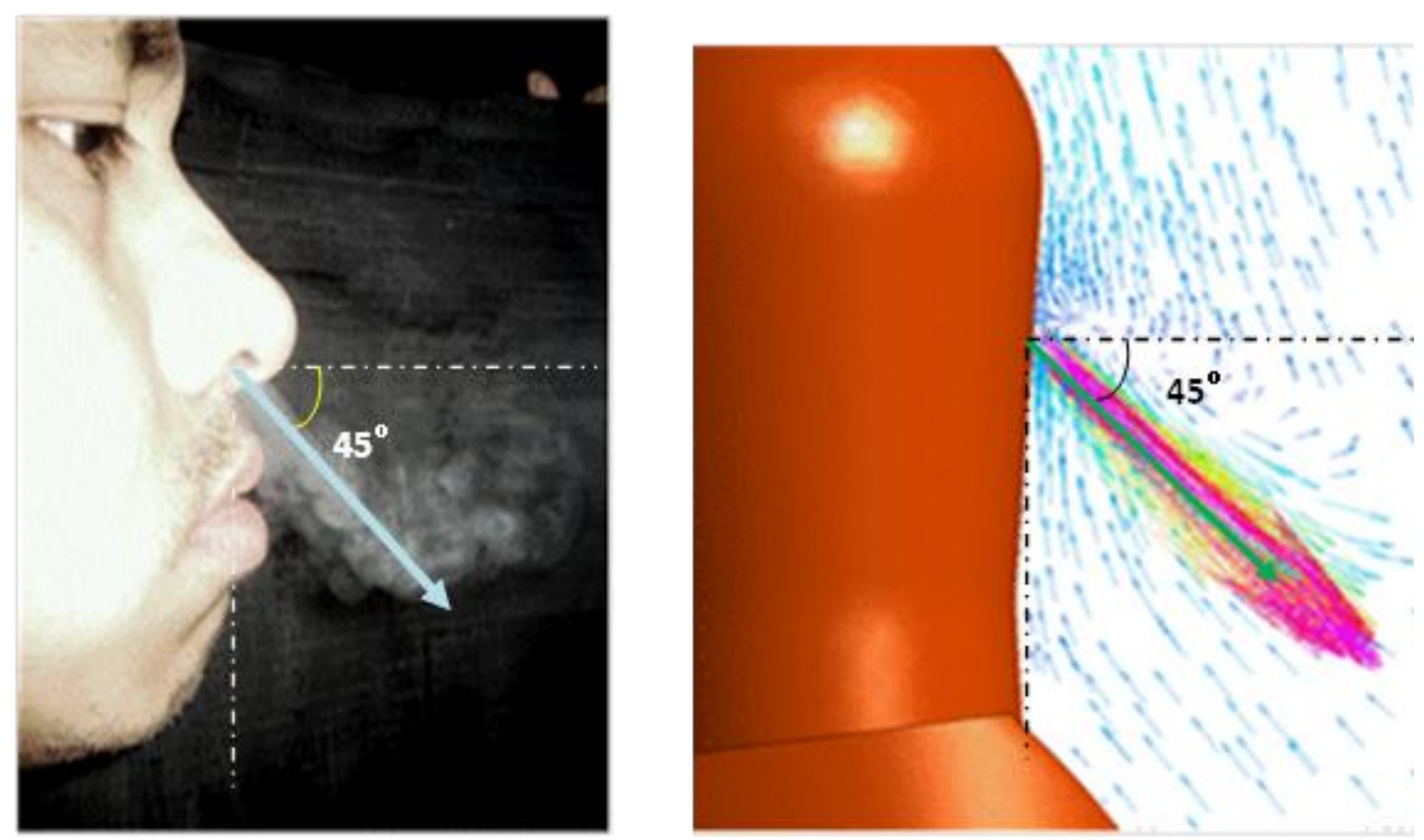

Figure 4 Flow field with constant exhalation (measurement) (a) physical visualization

(b) Velocity-vector (CFD)

\section{2: Grid Development and Sensitivity Test (Step 2)}

The mesh volume generated at the location of the outlet is presented in Figure 5. In the near-wall regions, boundary layer effects give rise to velocity gradients that are greatest normal to the wall. Computationally efficient meshes in these regions (i.e. wall regions) require that the elements have high aspect ratios. If tetrahedral elements are used, then a 
prohibitively fine surface mesh may be required to avoid generating highly skewed tetrahedral elements at the face. CFX-Mesh overcomes this problem by using prisms to create the mesh that is finely resolved normal to the wall, but coarse parallel to it. In the case of the circular outlet, this was achieved by giving an edge sizing to the circumference as illustrated in Figure 5. The Expansion Factor used for this model was 1.2 (i.e. each successive layer, is approximately 1.2 times thicker than the previous one as it moves away from the face to which the inflation was applied). Also, inflation is added to help resolve large gradients that can occur in the boundary layers that form on walls (both in temperature or velocity).

In addition, because the size of the nose at the surface of the CSP face is extremely small, the difference between the nose geometry and the face surface geometry is comparatively huge which makes the solution difficult to converge. A finer mesh would reduce the number of time steps necessary to reach a solution and reduce the spatial and temporal discretisation errors especially when a transient condition is applied as in this study.

The mesh sensitivity was ascertained by analysing the change in the results with different grid sizes. The grid refinement involved:

- Increasing the density of the grid at the walls.

- Increasing the density of the grid on the human manikin.

- Increasing the density of the grid on the heat sources

- Increasing the density of the grid at the inlet and outlet. 


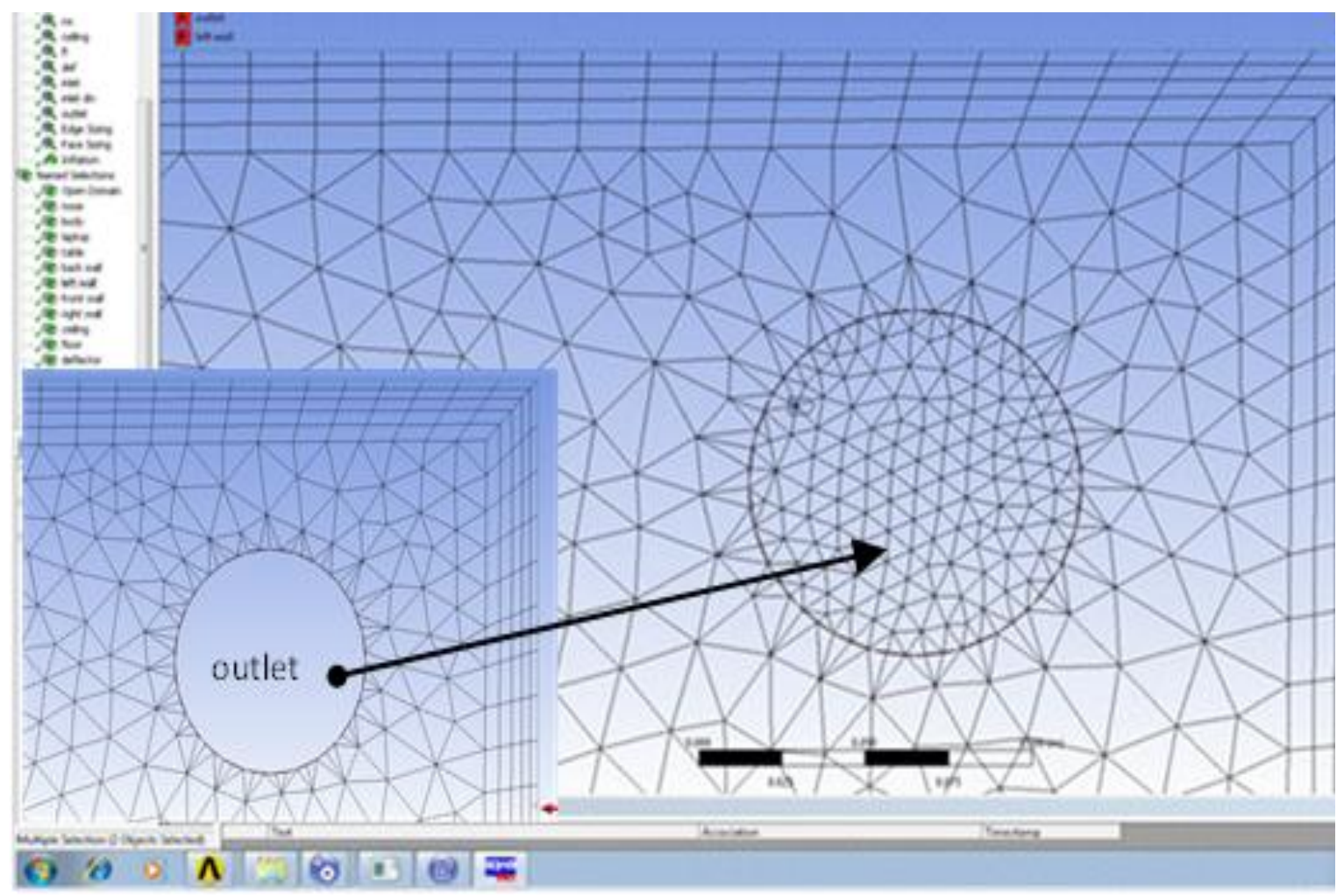

Figure 5 Fine mesh for a circular outlet near-wall region.

Based on these changes a grid comparison (coarse, medium and fine mesh) was carried out to justify the numerical results obtained from the simulation. To investigate these factors, a steady-state simulation was performed for all three grids with the flow field computed each with twice the number of grid points in $\mathrm{X}, \mathrm{Y}$ and $\mathrm{Z}$ direction. From the results of the descriptive analysis, the average air temperature at all locations, including the outlet, were selected to compare the results from the different grid sizes with the experimental data as illustrated in Figure 6. It is observed that the median of the air temperatures using the fine and medium mesh as well as data obtained from the experiment is comparable $\left(20.7^{\circ} \mathrm{C}\right)$. However, the standard deviation and the range between the inter-quartiles predicted for the medium mesh and the coarse mesh were relatively large compared to the fine mesh and the experimental results. The median value with the coarse mesh was under estimated $\left(\sim 20.4{ }^{\circ} \mathrm{C}\right)$. It is clear that the fine grid 
(with 1,680,419 total number of elements) produced more sensible results that agreed with experimental values and therefore, will be used for further CFD solutions in this research.

Further to the above mentioned results, Table 3 shows a comparison between the predicted and the measured temperatures in the plume above the manikin $(0.2 \mathrm{~m}$ and 0.4 m above the manikin's head) for all three grid sizes. Results indicate that the coarse mesh underestimated the values for the velocity and temperature profiles. The medium mesh is also observed to underestimate the temperature values but overestimate the air velocity values. As expected, the CFD results for both velocity and temperature profiles

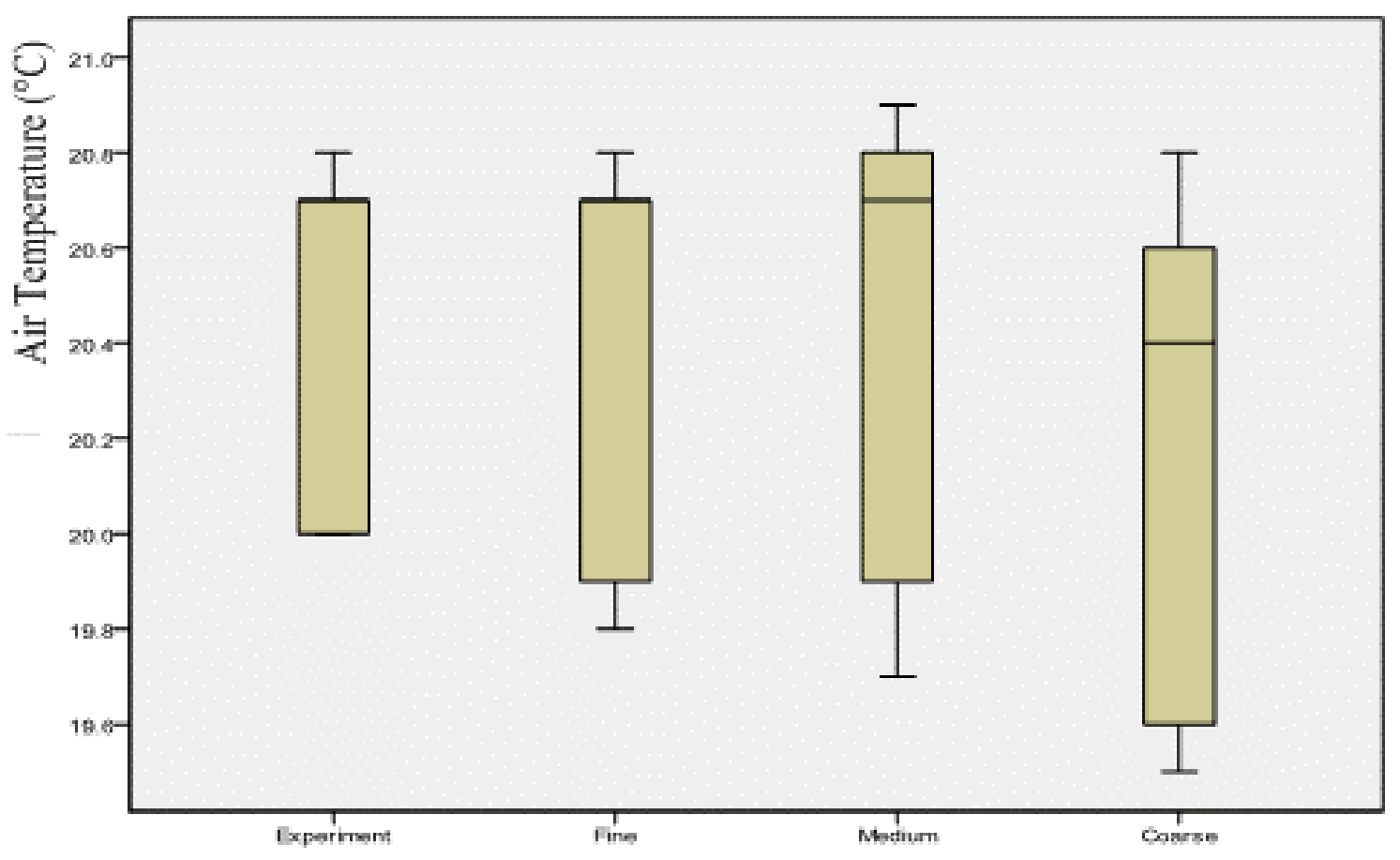

Figure 6 Comparisons of temperature results for different grid sizes: Air temperature plot predicted for the fine mesh are in good agreement with those from the measured data. 
Table 3 Velocity and temperature differences for different grid sizes above the manikin's head (1.2 $\mathrm{m}$ and $1.4 \mathrm{~m}$ from the floor)

\begin{tabular}{|ll|l|l|l|l|}
\hline & & Experiment & Coarse mesh & Medium mesh & Fine mesh \\
\hline Temperature $\left({ }^{\circ} \mathrm{C}\right)$ & $1.4 \mathrm{~m}$ & 22.2 & 20.8 & 21.8 & 22.1 \\
\hline & $1.6 \mathrm{~m}$ & 21.3 & 20.5 & 20.9 & 21.3 \\
\hline Velocity $\left(\mathrm{ms}^{-1}\right)$ & $1.4 \mathrm{~m}$ & 0.12 & 0.07 & 0.15 & 0.12 \\
\hline & $1.6 \mathrm{~m}$ & 0.13 & 0.09 & 0.16 & 0.13 \\
\hline
\end{tabular}

In addition the finer mesh was observed to converge with fewer iteration steps (263 iterations). This result also shows that the finer mesh would reduce the time steps necessary to reach a solution and reduce the spatial and temporal discretisation errors especially when a transient condition is applied.

\section{3: Numerical Simulations using Three Different turbulence Models (Step 3)}

Based on the grid sensitivity test, it is concluded that the fine mesh resolution provides a suitable solution for the intended simulations. In CFX (which is the main package of CFD models used in this research) there are various turbulence models available for general simulation purposes. These models were accepted by the researchers to be the most appropriate for the applications that are being considered. These models include a zero-equation model (0-eq) (Chen and Xu 1998), a Low Reynolds number $k-\varepsilon$ model (LRN $k-\varepsilon$ ) (Launder and Spalding 1974) an RNG $k-\varepsilon$ model (RNG) (Zhang et al. 2005) an Shear Stress Turbulence k-omega (SST $k$ - $\omega$ ) model (Menter, Kuntz, and Langtry 2003), a large-eddy-simulation model (LES) (Jouvray and Tucker 2005) and a few others. The complicated nature of turbulent flow characteristics in enclosed 
environments pose significant challenges on boundary conditions which influence the use of turbulence models(Ferrey and Aupoix 2006). As a result, it is always difficult to judge the outputs after simulation. Therefore, in order to ascertain which turbulence model is appropriate for this study, it is necessary to consider three models (RNG k- $\varepsilon$ model, Standard k- $\varepsilon$ (SKE) model and SST k- $\omega$ model) and monitor the results in detail to evaluate the simulated flows within the chamber.

\subsection{1: Smoke Test}

It is important to understand the general flow patterns around the room before studying the effect of turbulence models. However, deciding which turbulence model is best suited, a test involving smoke injection at the air inlet was carried out to visualise the flow pattern within the chamber. A smoke generator was used to inject smoke at the inlet with specified air supply rate. Snapshots were taken to monitor the movement of the smoke along the ceiling plane. Figure 7 shows the smoke distribution along the chamber ceiling. As the flow hits the supply plate (at the inlet) it creates a ceiling attachment zone due to the Coanda effect (following the path illustrated by the white arrows). This flow is drawn vertically by vortex (zone 1), travels along the ceiling plane (zone 2) and later separates (zone 3) at a jet separation point of approximately $2.13 \mathrm{~m}$ from the inlet wall. This separation is observed to take place due to the effect of gravity or the lower pressure at the opposite wall. The relationship between the flow field and Coanda effect is investigated in this study.. 


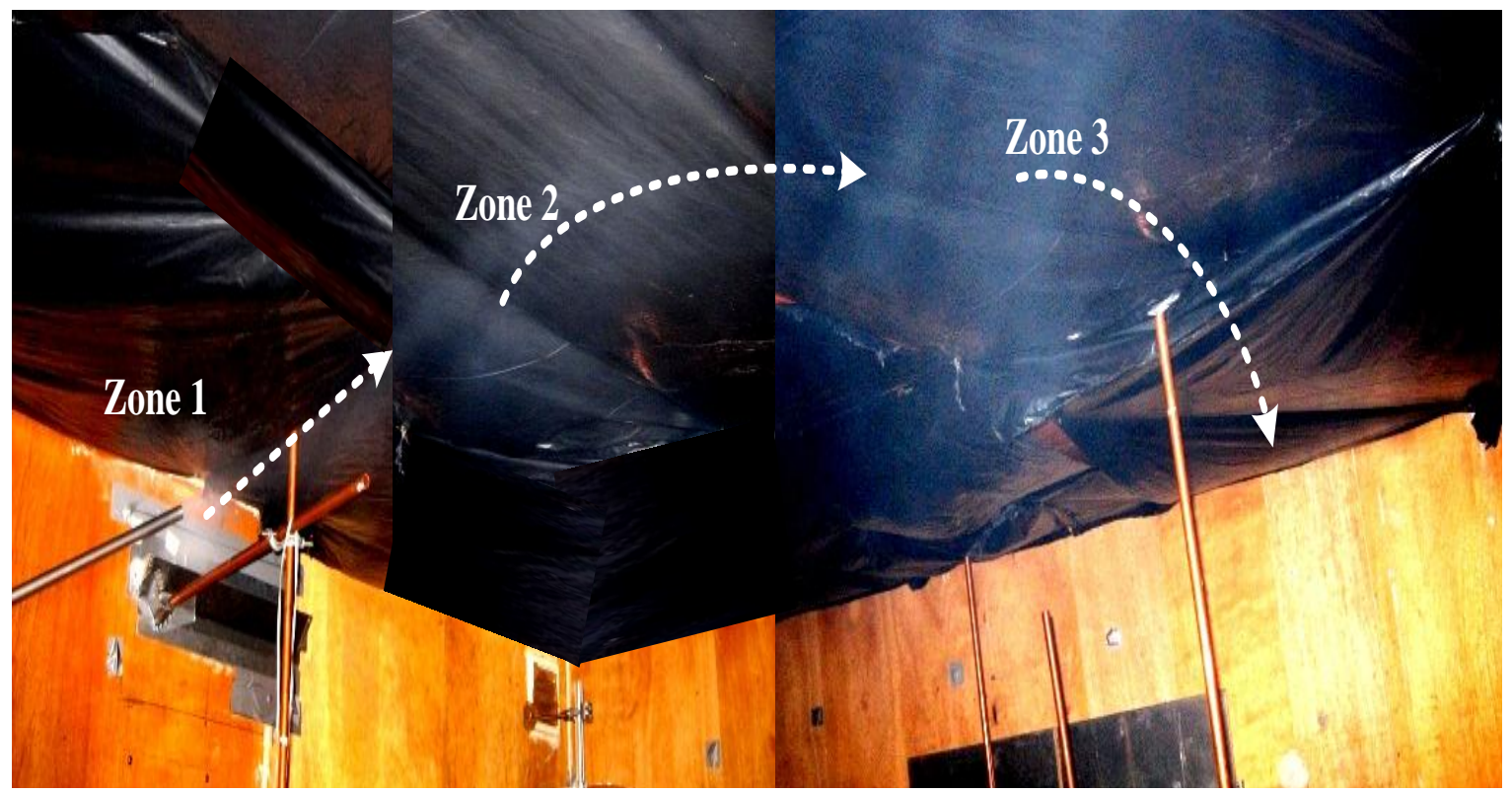

Figure 7 Smoke visualisation test showing the flow from the inlet supply.

The verification of the general air flow pattern from the supply inlet over the ceiling is presented in Figure 8 using CFD. This effect is modelled for comparison and analysis using the three turbulence models. The models are compared with reference to the jet separation point $(y=2.13 \mathrm{~m})$ which was obtained from the smoke tests. From the simulations, it was observed that the jet separation point for both RNG model and the SST model are in agreement with that of the smoke test. The jet is seen to separate from the ceiling at about the same point as in the smoke test. However, a larger vortex can be seen to the left of this separation point for the SST model distorting the flow path downstream, while in the RNG model, the spreading out of the flow continues smoothly towards the wall opposite the inlet wall (right side of the manikin). In this case, a smaller vortex appears at the lower region of that wall with low air velocity observed. 


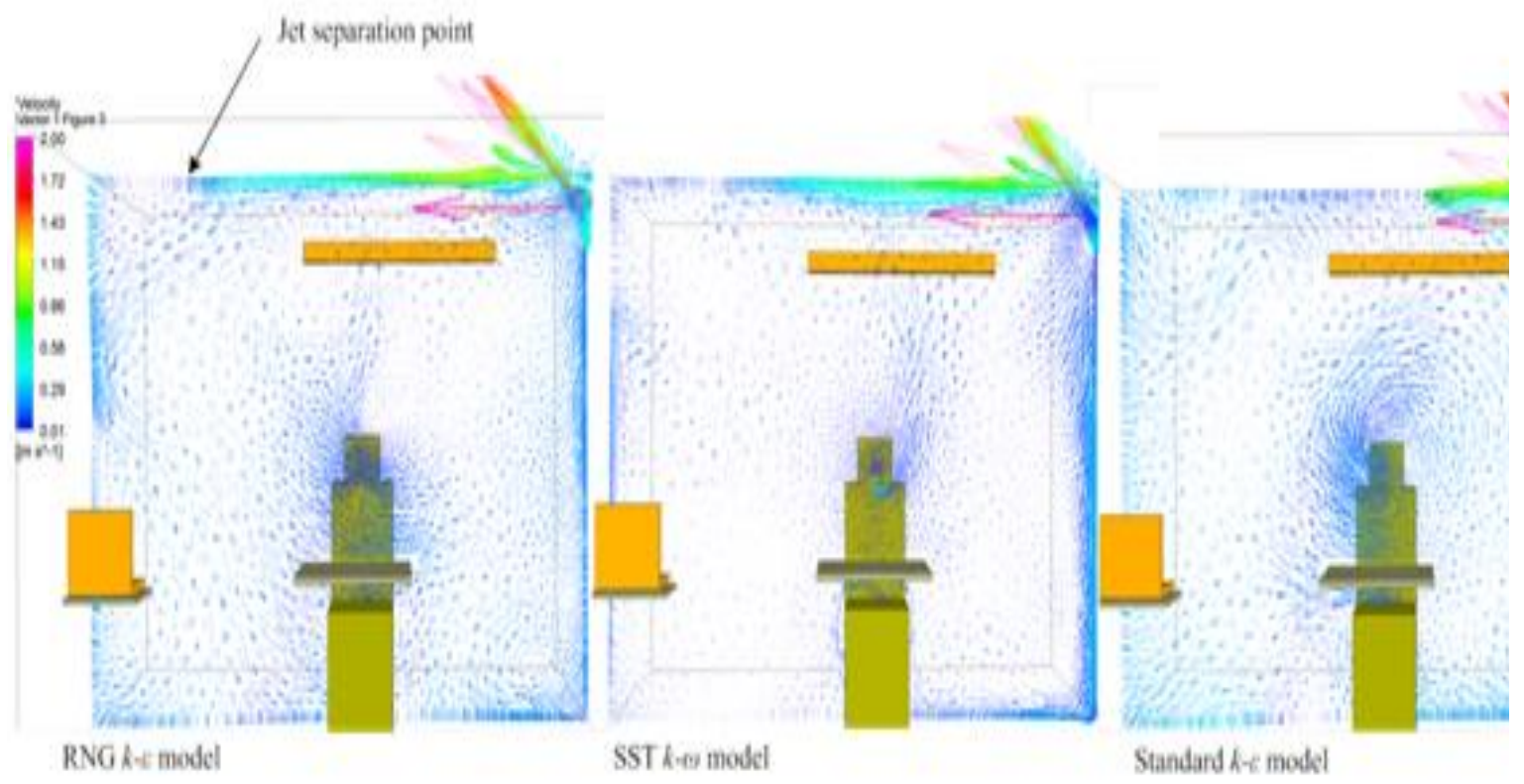

Figure 8 Velocity flow fields from the inlet supply demonstrating the supply jet across the $\mathrm{Y}$ plane (ceiling).

Unlike the results obtained using the RNG and the SST model, the magnitude of the velocity vectors using the SKE model is observed to be reduced much earlier at approximately $1.3 \mathrm{~m}$ from the inlet wall. However, the jet separation point is noted to be similar to the other two models but with a larger vortex forming at the upper region towards the corner of the opposite wall. The intensity (i.e. magnitude) of the velocity vectors in the chamber is also observed to be higher using this model. A possible reason for this would be that this type of turbulence model does not predict the localised turbulence effects very accurately, e.g. buoyancy effect, obstructions, etc. Based on the results illustrated above, it can be concluded that the flow stratification predicted using the RNG $k-\varepsilon$ model shows better agreement with the smoke test measurements and that the SKE model was the worst. This results coincides with the findings from Sekhar and Willem (2004) whom successfully used the RNG $k$ - $\varepsilon$ model to study flow patterns in a large office area. This model performed better than the standard $k-\varepsilon$ model for a mixed 
convection flow case and an impinging jet. In addition, Zhang et al. (2007) successfully conducted a comprehensive validation of the RNG $k$ - $\varepsilon$ model for air distributions in a quarter of a classroom, an individual office and a cubicle office and with a displacement ventilation system. The results of the computed temperature and velocity validations correlated reasonably well with the measured data.

\section{4: Verification and Validation (Step 4)}

As with most modelling packages, verification and validation is of great importance and within the field of CFD analysis in particular. For verification, the physical parameters, which are of importance in this study, are first identified. The variables such as airflow and temperature in the room need to be investigated.

The validation procedure for assessing the accuracy of the three different turbulence models when compared to the real physical situation would be considered in the next section. This is also to determine the representative degree of accuracy of the CFD solutions compared with that of the measurements carried out in the chamber (i.e. focusing on the indoor temperature and velocity flow profiles).

\subsection{1: Indoor Air Temperature}

Figure 9 illustrates the comparison of the chamber's spatial distributions of indoor air temperature. The figure shows the comparison between the experimental and numerical results for the turbulence models RNG, SKE and SST. The layout of sensors and the 3D computation model, illustrated in a plan view is shown in the insert for Figure 9, to show the height of sampling sensors at different locations (identified by yellow markers). The coordinates of these monitoring sensors is listed in Table 1 and Figure 1. 


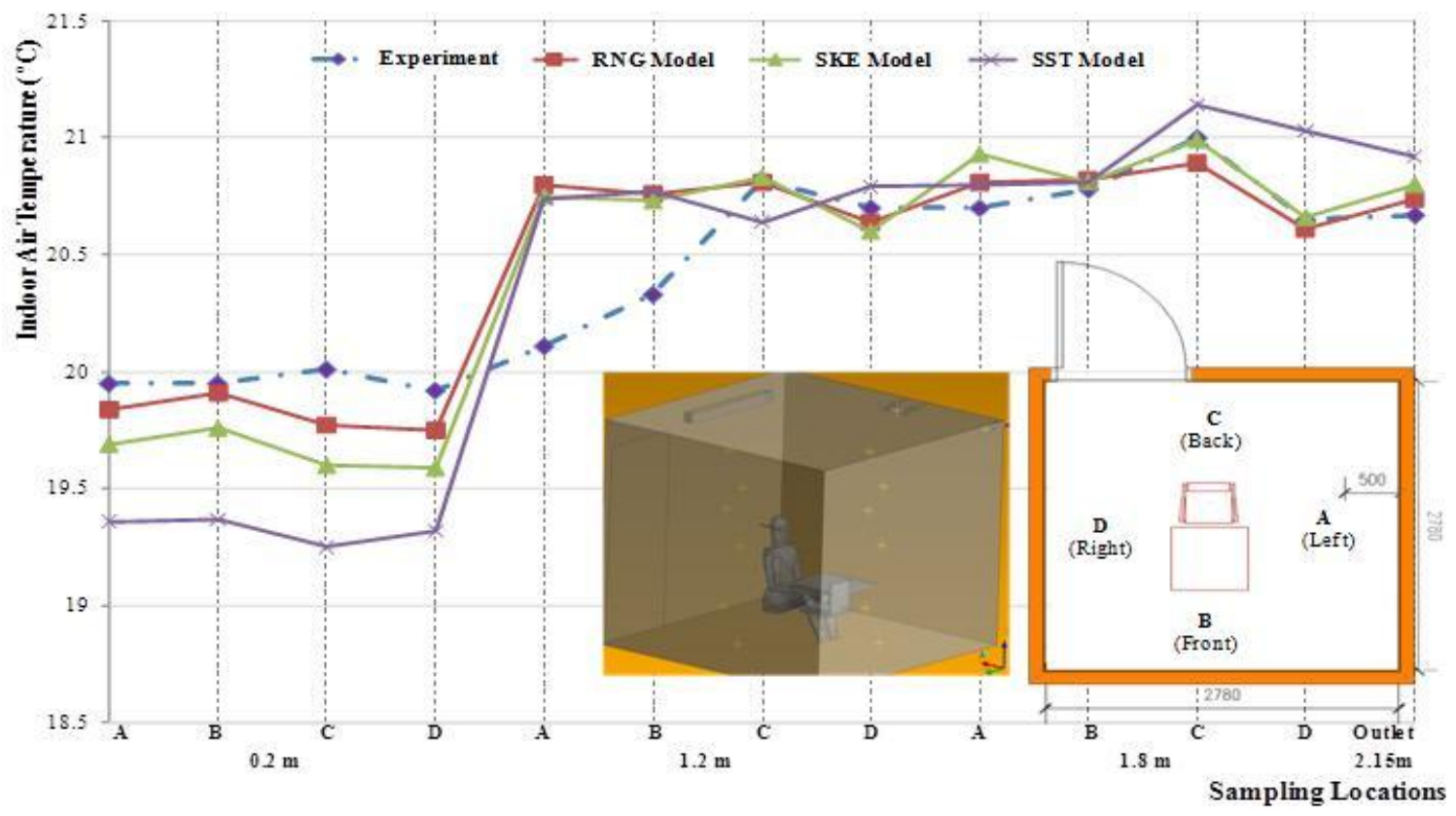

Figure 9 Comparison of the spatial distributions of indoor air temperature in the chamber:

Experiments and numerical simulations

Based on this figure, it can be clearly seen that the RNG model better predicts indoor temperatures for the lower regions of the chamber (i.e. $0.2 \mathrm{~m}$ height from the floor), whereas the SKE and SST models showed larger variations in the simulated values compared to the experimental values for this region. Nevertheless, some degree of agreement between all three models was observed for the higher regions (i.e. $1.2 \mathrm{~m}$ and $1.8 \mathrm{~m}$ ), and these results were comparable although they show some overestimations of temperature values at location A (left) and B (front) at $1.2 \mathrm{~m}$ height (i.e. less than $1.0^{\circ} \mathrm{C}$ ).

The overestimation of this temperature gradient in Figure 9 may have been due to the Coanda effect (i.e. close to the jet separation region) and the interaction with the plume from the thermal manikin. Taking the steady-state value of temperature at each monitoring point, the temperature stratifications were observed to be significant in the 
SST turbulent model especially at the lower regions. Generally, the SST model predicted lower temperatures in the lower region of the chamber leading to a sharper gradient to the upper regions of the chamber.

In addition, the temperature stratifications of buoyant plumes above the manikin's head can be observed for all three models. This is supported in studies conducted by $\mathrm{Li}$, et al.(2013), where they state that the thermal plume actually develops along the body surface from the lowest body segments such as the feet. Sorensen and Voigt (2003) compared CFD calculations to measurements by PIV of a nude manikin, in both cases care was taken to ensure set-ups took into account all relevant conditions. The CFD results were validated and compared to particle image velocimetry (PIV) measurements. The results showed satisfactory agreement, although they cited a slightly higher velocity was apparent in the CFD output. Though this analogue is drawn, it must be noted that PIV was not used in this study

As illustrated in Table 4, the thermal stratifications using the SST turbulence model are observed to be greater compared to the other two models. When observing the verification of the thermal plume above the manikin's head (i.e. $1.4 \mathrm{~m}$ and $1.6 \mathrm{~m}$ height above the floor), the predictions of the temperature values by both SKE and SST models did not correspond to that of measured data.

Table 4 Experimental and predicted values of air temperature above the human manikin head height

\begin{tabular}{|l|l|l|}
\hline $\begin{array}{l}\text { Measurement height } \\
\text { Comparison }\end{array}$ & $\begin{array}{l}\mathbf{1 . 4} \text { m height } \\
\left({ }^{\circ} \mathrm{C}\right)\end{array}$ & $\begin{array}{l}\mathbf{1 . 6} \mathbf{~ m} \text { height } \\
\left({ }^{\circ} \mathrm{C}\right)\end{array}$ \\
\hline Experiment & 22.2 & 21.3 \\
\hline RNG $k-\varepsilon$ model & 22.1 & 21.3 \\
\hline SKE model & 21.6 & 21.2 \\
\hline SST $k-\omega$ model & 22.9 & 22.6 \\
\hline
\end{tabular}


The SST model calculated significantly higher temperature values while SKE model on the other hand, is observed to under-estimate the temperature value at $1.4 \mathrm{~m}$ height with a difference of $0.6 \mathrm{~K}$. As expected, the predicted results for the thermal plumes above the manikin's head obtained using the RNG model is observed to correspond better with the experimental results. Therefore, in this case, the turbulence model that predicted the thermal plumes closest to the experimental results was the RNG model.

\subsection{2: Air Velocity Flow Fields}

A further investigation is the airflow profiles in the chamber to help understand the patterns of airflow and velocity within the enclosed environment. Similar to Figure 9 for temperature, the velocity field at several locations within the chamber is presented in Figure 10. Unlike the temperature plot, the numerical results from the three turbulence models did not correspond well to that of experimental results. Although, there is a good correlation between predictions using the RNG model and measurement, it was observed that RNG and the SKE model have over predicted most of the velocity values. Conversely, the SST model has under predicted most of the results except for location C at a height of $1.8 \mathrm{~m}$. It is also worth mentioning that the measurement of low velocities

$\left(<0.1 \mathrm{~ms}^{-1}\right)$ is not very reliable which could have been a cause for the deviation with the predictions. Most indoor environments have low mean air velocity and therefore, the Reynolds number, Re, is also generally low $\left(\sim 10^{5}\right)$ which may implies that aspects of the flow would either be laminar or transitional. Where transitional flows are involved, they can be sensitive to the details of the implementation of boundary conditions, numerical schemes, and differences in physical models. Hence, it is difficult to identify the cause 
of possible discrepancies if the SST model is to be used further in this study. On the other hand the RNG model tends to better predict the flow in low Reynolds number and that buoyancy-driven flows better than the other models tested (Zhai et al. 2007)

Considering that this study has emphasis on the spatial distribution of the flow fields within the chamber, it is more significant to assess the general flow pattern instead of the absolute velocity values.

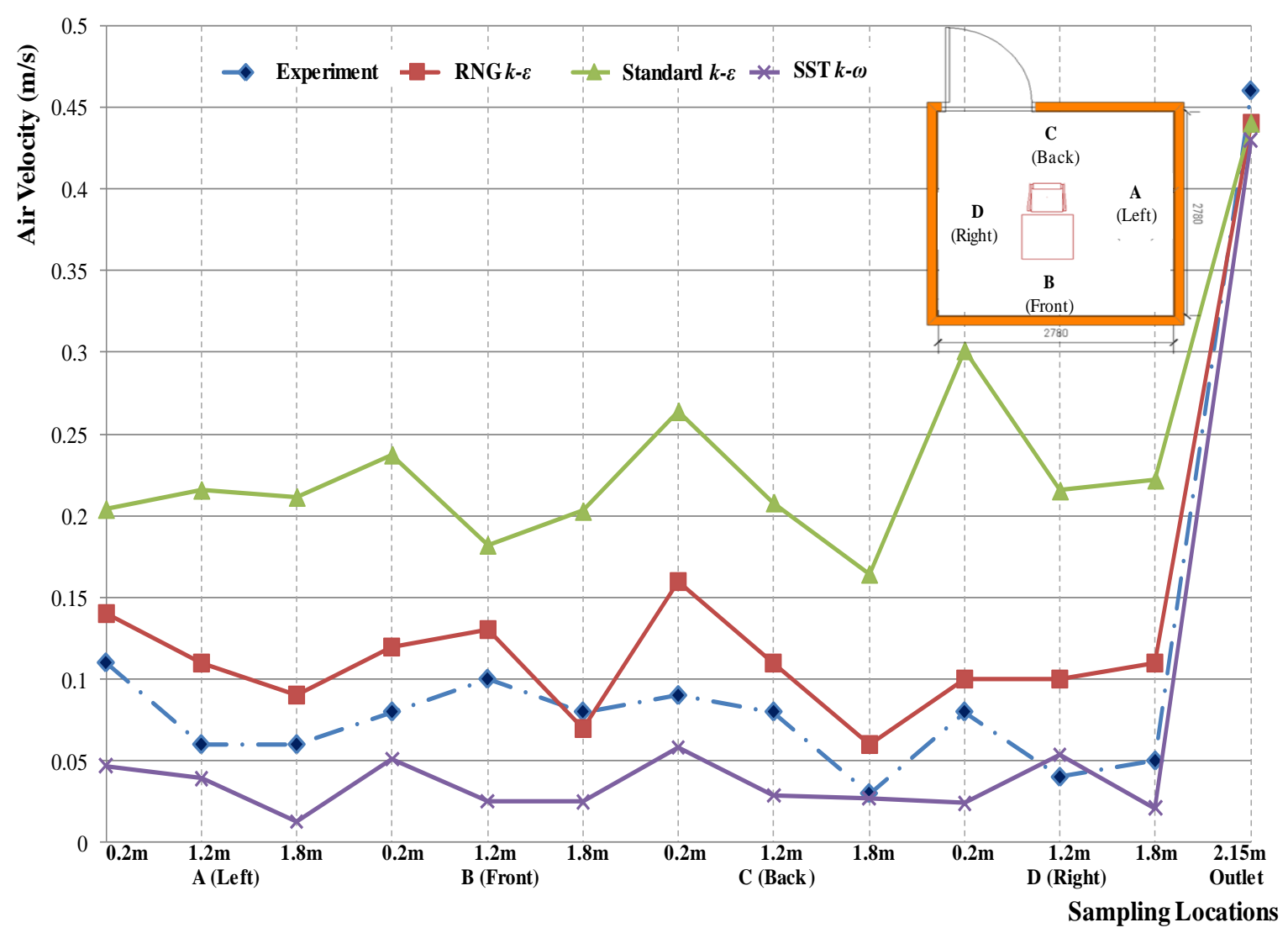

Figure 10 Comparison of air velocity profiles in the chamber: experimental and numerical simulations.

As for the temperature profiles of the plume above the manikin, the predicted results of the air velocity using the RNG model was observed to correspond better with the experimental results, as listed in Table 5. The buoyant plumes for the SST model overestimated the velocity flow at $1.4 \mathrm{~m}$ height above the floor with a significant different of $0.13 \mathrm{~ms}^{-1}$ from the experimental measurement. At $1.6 \mathrm{~m}$, both the SST and 
SKE model overestimated the air velocity with a difference of about $0.05 \mathrm{~ms}^{-1}$ compared to the measured values.

Table 5 Experimental and predicted values of air velocity above the human manikin head height

\begin{tabular}{|l|l|l|}
\hline $\begin{array}{l}\text { Measurement height } \\
\text { Comparison }\end{array}$ & $\begin{array}{l}\mathbf{1 . 4} \text { m height } \\
\left(\mathrm{ms}^{-1}\right)\end{array}$ & $\begin{array}{l}\mathbf{1 . 6} \mathbf{~ m} \text { height } \\
\left(\mathrm{ms}^{-1}\right)\end{array}$ \\
\hline Experiment & 0.12 & 0.13 \\
\hline RNG $k$ - $\varepsilon$ model & 0.12 & 0.13 \\
\hline SKE model & 0.15 & 0.18 \\
\hline SST $k-\omega$ model & 0.25 & 0.18 \\
\hline
\end{tabular}

\section{5: Selection of the Manikin Shape (Step 5)}

The modelling and prediction of a computer simulated person (CSP) has been the subject of contemporary research. Previous studies have simulated from very simple to very complex shapes for CSP (Murakami, Kato, and Zeng 2000, Kilic and Sevilgen 2008, Sørensen and Voigt 2003, Gao and Niu 2005). Various shapes of CSPs ranging from a very detailed human model (manikin) to a simplified 3D shape of a box representation (boxman) were developed and widely adopted in both research and industry to study and provide more insight into thermal comfort issues. These models are roughly put into three categories: simplified shapes (Murakami, Kato, and Zeng 2000, Al-Mogbel 2003, Xing, Hatton, and Awbi 2001), standard human manikins (male and female) (Sørensen and Voigt 2003, Tanabe et al. 2002) and realistic models (Yang et al. 2007, Treeck et al. 2009, Zhang and Yang 2008). For simplicity of the CFD modelling, a box human shape would be used for CFD validation test (Figure 11a) while a more realistic shape of a male body (Figure 11b) will be used in the transient simulation test in the chamber. 

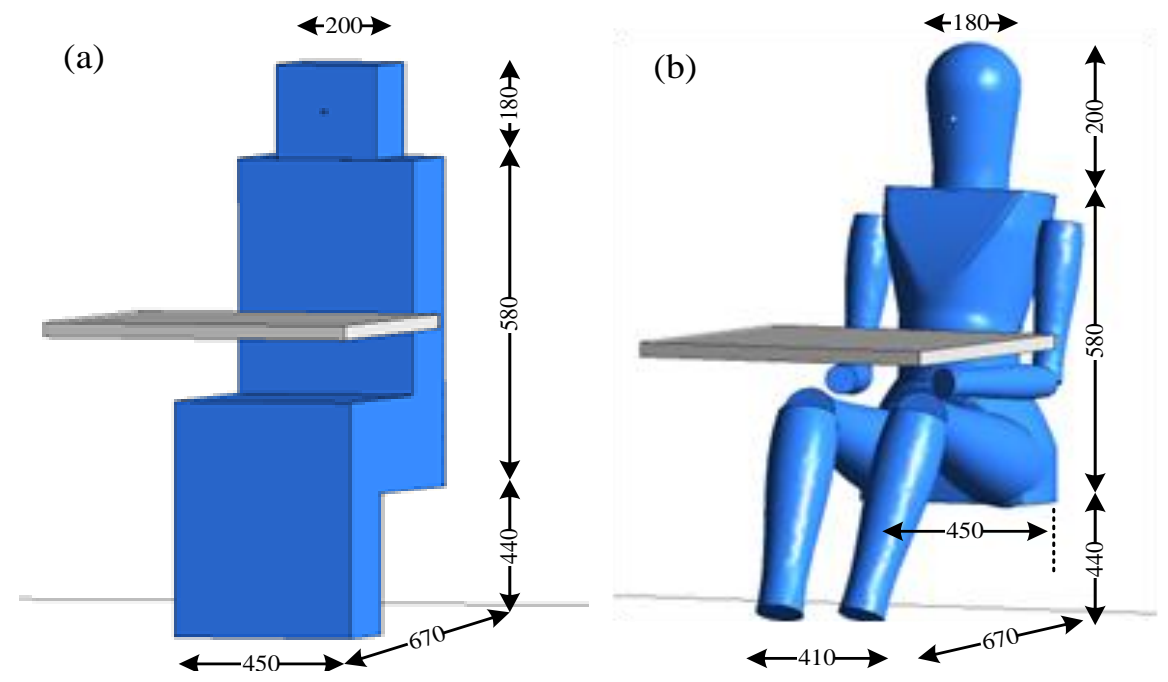

Figure 11 Two CFD models of a seated human to compare. (a) boxman and (b) manikin. Measurements are in $\mathrm{mm}$.

In principle, the closer the simulated geometry is to the real geometry, the more accurate the predictions are. However, when the geometry is complex, it can be difficult to create a mesh. Therefore, the hands and feet of the manikin were not included and this eliminated the need to mesh the individual fingers, which requires the use of very small elements and thus increase the computational time. In both models, an unstructured grid was used. Prismatic cells were used near the solid surfaces to resolve the wall jet and the thermally driven flow near the body of the person. A slightly finer grid was used in the region above the shoulders of both CSPs. The manikin model is discretised into $2,763,482$ cells while the boxman is discretised into $1,680,419$ cells Although the element size in both the boxman and manikin models was the same, the total number of elements was almost double in the manikin model due to the complexity of the geometry. 


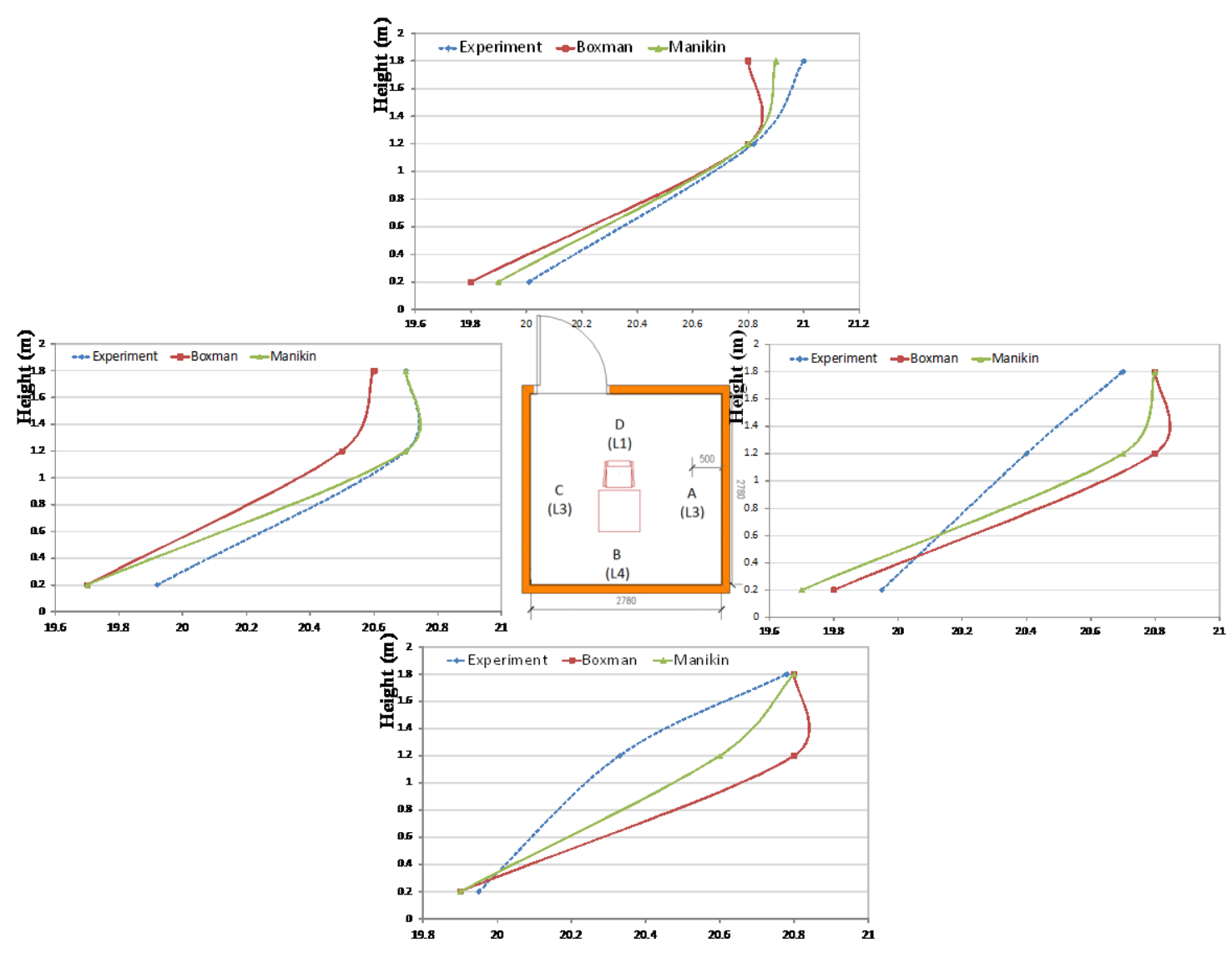

Figure 12 CFD predicted temperature profiles at $0.5 \mathrm{~m}$ away from the side walls for both CSPs.

For the temperature profiles in the chamber using both CSPs are observed to correspond well with the experimental results across all locations. However, significant differences between the CFD results using both CSP and the measured data were observed at location $\mathrm{B}$ and location A, specifically at $\mathrm{z}=1.2 \mathrm{~m}$. The differences that occur in location $\mathrm{B}$ and $\mathrm{A}$ using the boxman are $0.5 \mathrm{~K}$ and $0.4 \mathrm{~K}$ respectively, while with the manikin model, the differences were only $0.3 \mathrm{~K}$ at both locations. This is probably due to the complexity of the geometry which further increases the number of cells especially at the face area of the manikin. As a result, the CFD predictions using the manikin have yielded much smaller differences with measurements compared to the boxman. 
Figure 12. CFD predicted temperature profiles at $0.5 \mathrm{~m}$ away from the side walls for both CSPs.

At the upper $(\mathrm{z}=1.8 \mathrm{~m})$ and lower regions $(\mathrm{z}=0.2 \mathrm{~m})$, reasonable predictions are obtained from both CSP models. A maximum difference between the boxman and the measured data is $0.3 \mathrm{~K}$ at both these heights, while with the manikin a difference of $0.1 \mathrm{~K}$ and $0.2 \mathrm{~K}$ are obtained at the upper and lower regions respectively. At the upper region, the heat plumes above the CSP's head would have caused a natural convection flow that resulted in an increase in the air mixing similar to the experimental results. These findings indicate that the CFD predictions using the manikin model have better agreement with measured data.

To verify the overall flow filed in the chamber, the predicted simulations of the (a) temperature contours and (b) velocity contours for the different CSP geometries are compared in Figure 14. These contour plots are generated along the symmetry plane of L2 (as in Figure 13). The velocity is based on the inlet air supply of $2.0 \mathrm{~ms}^{-1}$.

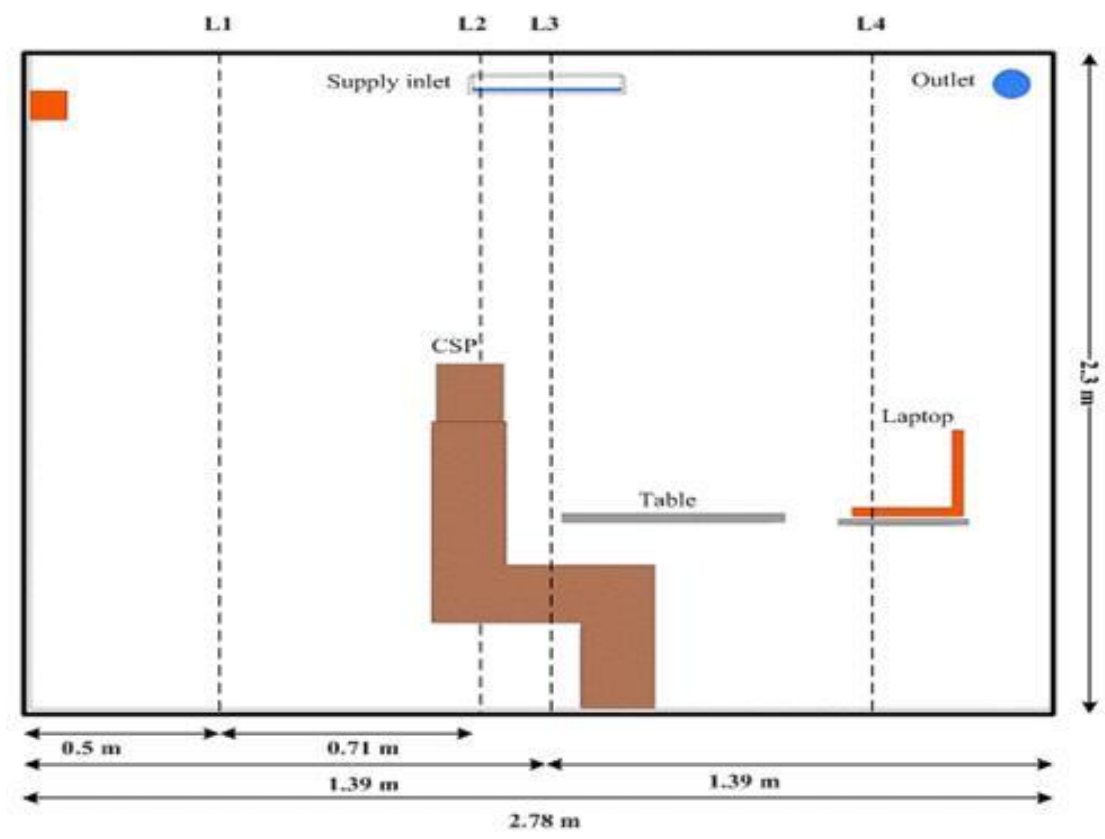

Figure 13 Location of measured velocity and temperature profiles 


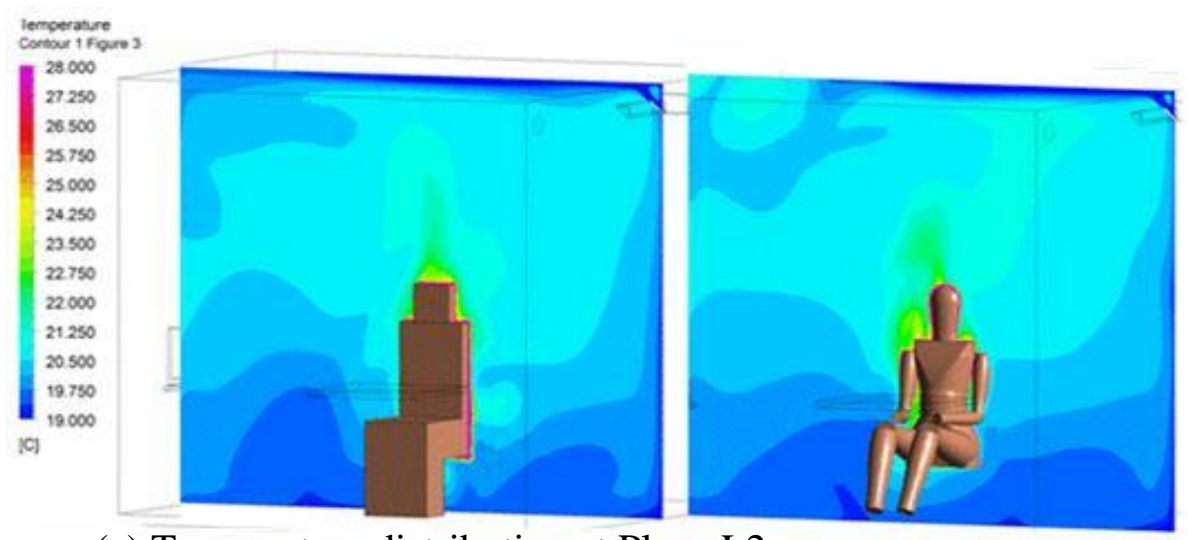

(a) Temperature distribution at Plane L2

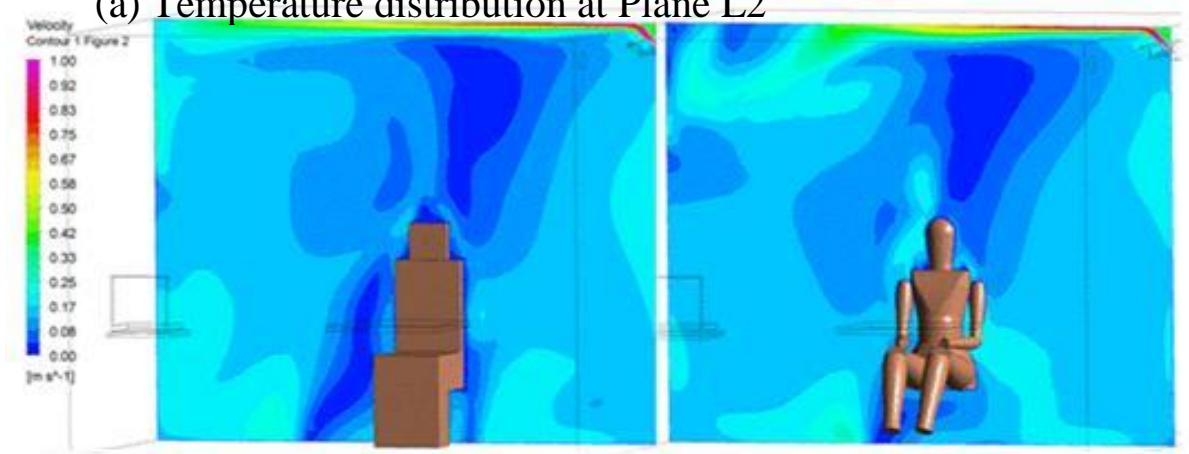

(b) Velocity distribution at Plane L2

Figure 14 Air flow field around the seated CSP

Similar to the results plotted in Figure 12, the temperature contours in Figure 14(a) appear to have relatively minor differences across the vertical plane. The heat from the CSP causes a thermal plume to form above its head. It also causes a stratified layer with warmer, less dense fluid in the upper part of the room. However, a slight increase in air temperature around the manikin's shoulders and head was observed when compared to the boxman. This was probably due to the more detailed geometry and grid complexity around these surfaces.

In Figure 14(b), the results of the velocity contours on the left hand side and close to the inlet, return quite similar velocities for both CSPs whilst the flow field between the two CSPs is slightly different on the right hand side. Due to low airflow environment $\left(<0.1 \mathrm{~ms}^{-1}\right)$, strong buoyancy may occur and fluid flows with strong buoyancy effects can be difficult to predict correctly when using a simplified CSP. In 
general the CFD predictions using both CSPs correspond well with the experimental results. However, the accuracy of the velocity and temperature profiles is better predicted using the manikin model.

\section{4: Conclusions}

The parameters needed to simulate the exhalation from a human were investigated in this paper using CFD. As demonstrated, proper simulation of nose and mouth geometry, i.e. flow exhalation, will be needed for studying the transport of exhaled air in a room. The research has provided comparisons between the results produced using three turbulence models (SKE, SST and RNG) with experimental results obtained in an environmental test chamber under controlled conditions. The parameters considered were air velocity, temperature and the buoyancy plume above the manikin's head. The following conclusions can be drawn:

- The RNG model simulation of the plume velocities above the manikin showed the closest agreement with the measured results. The difference between the average plume velocities at the measurement locations (i.e. $1.4 \mathrm{~m}$ and $1.6 \mathrm{~m}$ height above the floor at the centre of the manikins' head) was less than 0.01 ms-1. This difference was less than the experimental uncertainty of the air velocity sensor which was $\pm 0.02 \mathrm{~ms}^{-1}$. However, for the SKE model, the difference was $0.04 \mathrm{~ms}^{-1}$ and for the SST model was $0.09 \mathrm{~ms}^{-1}$. The temperature in the plume above the manikin's head (i.e. same measuring point as the plumes velocity) obtained using the RNG model also showed substantial agreement with the measured data. The difference is on average less than $0.1 \mathrm{~K}$, which was again less than the experimental uncertainty for the air temperature sensor, which was $\pm 0.15 \mathrm{~K}$. The difference in temperature using the SKE model was 
quite similar at about $0.06 \mathrm{~K}$ compared to the measured data. Conversely, the CFD results predicted using the SST model gives the highest discrepancy of 2.0

K.

\section{Acknowledgements}

The authors acknowledge the financial support from Ministry of Education Malaysia and University of Malaya, Malaysia.

\section{References}

Al-Mogbel, A. 2003. A coupled model for predicting heat and mass transfer from a human body to its surroundings. Paper read at Proceedings of the 36th AIAA Thermophysics Conference, Florida, USA.

Awbi, H.B. 2003. Ventilation of Buildings, 2nd edition. London: E \& FN Spon.

Bjorn, E., and P. V. Nielsen. 2002. "Dispersal of exhaled air and personal exposure in displacement ventilated rooms." Indoor Air no. 12 (3):147-64.

Buchanan, C.R. 1997. CFD Characterization of mechanically ventilated office room: the effect of room design on ventilation performance. Ph.D Diss, University of California, Irvine.

Chen, Q. 1995. "Comparison of different $k-\varepsilon$ models for indoor airflow computations." Numerical Heat Transfer no. 28 (Part B):353-369.

Chen, Q., and W. Xu. 1998. "A zero-equation turbulence model for indoor airflow simulation." Energy and Buildings no. 28 (2):137-144.

Deevy, M., Y. Sinai, P. Everitt, L. Voigt, and N. Gobeau. 2008. "Modelling the effect of an occupant on displacement ventilation with computational fluid dynamics." Energy and Buildings no. 40 (3):255-264.

Ferrey, P, and B Aupoix. 2006. "Behaviour of turbulence models near a turbulent/non-turbulent interface revisited." International Journal of Heat and Fluid Flow no. 27:831-837.

Ferziger, J.H, and M Peric. 2002. Computational Methods for Fluid Dynamics Berlin-Germany: $3^{\text {rd }}$ Edition (Revised), Springer.

Gao, N. P., and J. L. Niu. 2005. "CFD Study of the Thermal Environment around a Human Body: A Review." Indoor and Built Environment no. 14 (1):5-16. doi: 10.1177/1420326x05050132.

Gao, Naiping, and Jianlei Niu. 2006. "Transient CFD simulation of the respiration process and inter-person exposure assessment." Building and Environment no. 41 (9):1214-1222.

Gupta, Jitendra K., Chao-Hsin Lin, and Qingyan Chen. 2010. "Characterizing exhaled airflow from breathing and talking." Indoor Air no. 20 (1):31-39. doi: 10.1111/j.1600- 
0668.2009.00623.x.

Haselton, F. R., and P. G. Sperandio. 1988. "Convective exchange between the nose and the atmosphere." Journal of Applied Physiology no. 64 (6):2575-2581.

Holmes, S. A, A Jouvray, and P. G Tucker. 2000. An assessment of a range of turbulence models when predicting room ventilation. Paper read at Proceedings of Healthy Buildings 2000, at (2), 401.

Hyldgaard, C.E. 1994. Humans as a source of heat and air pollution. Paper read at Proc. of ROOMVENT 94, 4th international conference on air distribution in rooms. , at Krakow, Poland.

Jouvray, A, and P. G Tucker. 2005. "Computation of the flow in a ventilated room using nonlinear RANS, LES and hybrid RAN/LES." International Journal of Numerical Methods in Fluids no. 48 (1):99-106.

Karthikeyan, C. P., and Anand A. Samuel. 2008. "CO2-dispersion studies in an operation theatre under transient conditions." Energy and Buildings no. 40 (3):231-239.

Kilic, Muhsin, and Gökhan Sevilgen. 2008. "Modelling airflow, heat transfer and moisture transport around a standing human body by computational fluid dynamics." International Communications in Heat and Mass Transfer no. 35 (9):1159-1164.

Launder, B.E, and D.B Spalding. 1974. "The numerical computation of turbulent flows." Computer Methods in Applied Mechanics and Energy no. 3:269-289.

Li, Xiangdong, Kiao Inthavong, Qinjiang Ge, and Jiyuan Tu. 2013. "Numerical investigation of particle transport and inhalation using standing thermal manikins." Building and Environment no. 60 (0):116-125. doi: http://dx.doi.org/10.1016/j.buildenv.2012.11.014.

Lin, Zhang, T. T. Chow, C. F. Tsang, K. F. Fong, L. S. Chan, W. S. Shum, and Luther Tsai. 2009. "Effect of internal partitions on the performance of under floor air supply ventilation in a typical office environment." Building and Environment no. 44 (3):534545 .

Lin, Zhang, Lin Tian, Ting Yao, Qiuwang Wang, and T. T. Chow. 2011. "Experimental and numerical study of room airflow under stratum ventilation." Building and Environment no. 46 (1):235-244.

Loomans, Marcel Gerardus Louis Cornelius. 1998. The Measurement and Simulation of Indoor Air Flow. Ph.D Diss, Technical University, Eindhoven.

Lu, Tao, Anssi Knuutila, Martti Viljanen, and Xiaoshu Lu. 2010. "A novel methodology for estimating space air change rates and occupant $\mathrm{CO} 2$ generation rates from measurements in mechanically-ventilated buildings." Building and Environment no. 45 (5):1161-1172.

Mahyuddin, N, and H Awbi. 2010. "The spatial distribution of carbon dioxide in an environmental test chamber." Building and Environment no. 45 (9):1993-2001.

Mahyuddin, Norhayati. 2011. The Spatial Distribution of Carbon Dioxide in Rooms with Particular Application to Classrooms. Ph.D Diss, School of Construction Management and Engineering, University of Reading, Reading. 
McArdle, William D., Frank I. Katch, and Victor L. Katch. 2006. "Section 4: The Physiologic support systems." In Essentials of exercise physiology, 3rd edition. Lippincott Williams \& Wilkins.

Melikov, A., and J. Kaczmarczyk. 2007. "Measurement and prediction of indoor air quality using a breathing thermal manikin." Indoor Air no. 17 (1):50-59.

Menter, F.R., M. Kuntz, and R. Langtry. 2003. "Ten years of experience with the SST turbulence model." In Turbulence, Heat and Mass Transfer, edited by K. Hanjalic, Y. Nagano and M. Tummers. Redding, CT: Begell House Inc.

Murakami, Shuzo, Shinsuke Kato, and Jie Zeng. 2000. "Combined simulation of airflow, radiation and moisture transport for heat release from a human body." Building and Environment no. 35 (6):489-500.

Quatember, B. 2003. Human Respiratory System: Simulation of Breathing Mechanics and Gas Mixing Processes Based on a Non-Linear Mathematical Model. In International Conference on Health Sciences Simulation. Innsbruck, Austria.

Rim, Donghyun, and Atila Novoselac. 2009. "Transport of particulate and gaseous pollutants in the vicinity of a human body." Building and Environment no. 44 (9):1840-1849.

Sekhar, S. C., and H.C Willem. 2004. "Impact of airflow profile on indoor air quality-a tropical study." Building and Environment no. 39:255-266.

Shih, Yang-Cheng, Cheng-Chi Chiu, and Oscar Wang. 2007. "Dynamic airflow simulation within an isolation room." Building and Environment no. 42 (9):3194-3209.

Sørensen, Dan Nørtoft, and Lars Køllgaard Voigt. 2003. "Modelling flow and heat transfer around a seated human body by computational fluid dynamics." Building and Environment no. 38 (6):753-762.

Tanabe, S, Kobayashi K, Nakano J, Ozeki Y, and Konishi M. 2002. "A Comprehensive Combined Analysis with Multi-Node Thermoregulation Model (65mn), Radiation Model and CFD for Evaluation of Thermal Comfort." Energy and Buildings:637-646.

Topp, C, Nielsen PV, and DN Sørensen. 2002. "Application of computer simulated persons in indoor environmental modeling." ASHRAE Transactions no. 108 (2):1084-1089.

Treeck, Christoph van, Jérôme Frisch, Martin Egger, and Ernst Rank. 2009. Model-Adaptive analysis of indoor thermal comfort. Paper read at Eleventh International IBPSA Conference July 27-30, 2009-, at Glasgow, Scotland. pp 1374 -1381.

Vos, W., J. De Backer, A. Devolder, B. Partoens, P. Parizel, and W. De Backer. 2007. "Functional Imaging of Respiratoty System using Computational Fluid Dynamics." Journal of Biomechanics no. 40 (10):2207-2213.

Xing, H., A. Hatton, and H.B. Awbi. 2001. "A study of the air quality in the breathing zone in a room with displacement ventilation." Building and Environment no. 36:809-820.

Yang, Tong, Paul C. Cropper, Malcolm J. Cook, Rehan Yousaf, and Dusan Fiala. 2007. A new simulation system to predict human-environment thermal interactions in naturally ventilated buildings. Paper read at Proceedings of the 10th International Conference on Building Simulation (Building Simulation 2007), 3rd-6th September, at Tsinghua University, Beijing, China. Paper ID-424, pp. 751-756. 
Zhai, Z.Q., W. Zhang, Z. Zhang, and Q. Chen. 2007. " Evaluation of various turbulence models in predicting airflow and turbulence in enclosed environments by CFD: Part 1Summary of prevalent turbulence models." HVAC\&R Research no. 13 (6):853-870.

Zhang, L., T.T Chow, Qiuwang Wang, K.F Fong, and L.S Chan. 2005. "Validation of CFD model for research into displacement ventilation " Architectural Science Review no. 48 (4):305-316.

Zhang, Yi, and T Yang. 2008. Simulation of human thermal responses in a confined space. Paper read at 11th International Conference on Indoor Air quality and Climate, 17-22 August 2008, at Copenhagen, Denmark. pp ID: 875.

Zhang, Z., and Q. Chen. 2006. "Experimental measurements and numerical simulations of particle transport and distribution in ventilated rooms." Atmospheric Environment no. 40:3396-3408.

Zhang, Z., Z.Q. Zhai, W. Zhang, and Q. Chen. 2007. "Evaluation of various turbulence models in predicting airflow and turbulence in enclosed environments by CFD: Part 2Comparison with experimental data from literature." HVAC\&R Research no. 13 (6):871-886. 